\title{
Comportamento de secagem e explosão de concretos refratários com diferentes teores de água
}

\section{(Water content and the refractory castables' drying behavior)}

\author{
F. T. Ramal Jr, R. Salomão, V. C. Pandolfelli \\ Departamento de Engenharia de Materiais - DEMa \\ Universidade Federal de $S$. Carlos \\ Rod. Washington Luiz, km 235, C.P. 676, S. Carlos, SP 13565-905 \\ jr@polvo.ufscar.br,vicpando@power.ufscar.br
}

\begin{abstract}
Resumo
O crescimento do consumo de aço e da sua tecnologia de produção tem demandado proporcional desenvolvimento dos refratários, sendo umas das principais exigências o aumento de sua vida útil e a redução do tempo de parada dos equipamentos revestidos por esses materiais. Entre as diversas classes de refratários, os concretos vêm ganhando destaque nos últimos anos, principalmente por apresentarem facilidade de aplicação e reparo. Uma vez que esses materiais são aplicados na forma fluida, problemas no processo de secagem são freqüentemente encontrados na indústria. Assim, alguns trabalhos envolvendo aditivos de secagem têm surgido como forma de reduzir a ocorrência de explosões, sem a necessidade de diminuir drasticamente a taxa de aquecimento do refratário após sua aplicação. Entretanto, informações que relacionem a secagem com variáveis intrínsecas do processamento dos concretos ainda são escassas na literatura. Estudos reportam que a variação na quantidade de água influencia significativamente a reologia, e pode facilitar, por exemplo, a adição de fibras durante a mistura. Neste contexto, este trabalho teve como principal objetivo relacionar o teor de água com o comportamento de secagem e explosão de concretos refratários. Verificou-se que a adição de maior quantidade de água durante a mistura resultou em aumento da permeabilidade e queda na resistência a explosão dos concretos.

Palavras-chave: concretos refratários, teor de água, secagem, explosão.
\end{abstract}

\begin{abstract}
The growing technology employed on steelmaking industry resulted an equivalent development of refractories, where reductions of maintenance time and increasing working life have been one of the major requirements. The increase of monolithics consumption, especially castables, can be explained by their easy application and repair features. However, as the castables are applied in fluid form, problems with drying stage are frequently verified during this processing step. Studies in this area report the utilization of drying admixtures, which can avoid explosive spalling and optimize the drying rates. However, information about intrinsic variables, like water content, and its influence on the drying behavior of refractories castables are scarce in the technical literature. Thus, the objective of this work was to analyze the impact of water content on drying and explosive spalling behavior of refractory castables. The increase of water content resulted in castables with superior permeability and lower explosion resistance.
\end{abstract}

Keywords: refractory castables, water content, drying, explosion spalling.

\section{INTRODUÇ̃̃O}

Nos últimos anos, a utilização de refratários monolíticos tem sido impulsionada devido as vantagens apresentadas por esses materiais em relação aos pré-moldados tradicionais. Destacam-se entre elas a facilidade de aplicação e a não necessidade de conformação e queima prévias. Além disso, o contínuo desenvolvimento nessa área fez com esses materiais alcançassem propriedades termomecânicas similares aos pré-moldados tradicionais.

Por outro lado, a evolução tecnológica de suas propriedades têm resultado em materiais cada vez mais densos, e consequentemente menos permeáveis. Esse fato foi acompanhado por um aumento na dificuldade de secagem dos concretos, onde a aplicação de taxas de aquecimento inadequada pode levar a pressurização do vapor d'água dentro da estrutura e ocasionar a explosão do refratário; ou ainda elevar consideravelmente os gastos com energia e tempo de parada para manutenção de equipamentos. Desta forma, a adição de agentes de secagem, como o pó de alumínio [1] e as fibras poliméricas [2], surgiu para otimizar essa etapa do processo de produção do refratário, permitindo a utilização de ciclos de secagem mais agressivos.

No entanto, informações sobre a influência de variáveis intrínsecas do processamento no comportamento de secagem e explosão dos concretos ainda são escassas na literatura. Entre estas variáveis, pode-se citar o teor de água. Estudos relatam que o aumento no teor desse líquido influencia significativamente a reologia, facilitando, por exemplo, o transporte e aplicação de concretos bombeáveis [3]. Problemas 
na mistura ocasionados pela adição de fibras também podem ser minimizados aumentando-se o teor de água [4].

Além disso, a elevação na quantidade de água é responsável pelo aumento de permeabilidade e porosidade dos concretos [5], propriedades estas diretamente relacionadas com suas características de secagem, uma vez que altera a resistência mecânica, elevando com isso a tendência a explosão com o aumento da temperatura.

Desta forma, o presente trabalho teve como principal objetivo relacionar o teor de água utilizado no processamento de concretos refratários com seu comportamento de secagem e resistência a explosão. Para isso, foi utilizada como principal ferramenta a análise termogravimétrica, além da realização de medidas de permeabilidade, resistência mecânica e porosimetria de imersão (método de Arquimedes).

\section{MATERIAIS E MÉTODOS}

\section{Formulação e mistura}

A composição empregada no estudo foi formulada segundo o modelo de empacotamento de Andreasen [6], com coeficiente (q) 0,21 e diâmetro máximo de partícula igual a $4750 \mu \mathrm{m}$. A formulação consistiu de uma mistura de aluminas eletrofundidas (Alcoa alumínio Brasil) e calcinadas (Alcoa EUA), além de $2 \%(\%$ - $p)$ de cimento de aluminato de cálcio (CA 14 - Alcoa EUA). Ácido cítrico foi adicionado como dispersante na concentração de $0,26 \mathrm{mg} / \mathrm{m}^{2}$.

A mistura foi realizada em um reômetro para concretos, com adição de diferentes quantidades de água. Na Tabela I são especificados os teores de água utilizados, juntamente com seus respectivos valores de distância média de separação entre as partículas da matriz (IPS) e entre os agregados (MPT) [6]. Verificou-se em estudos anteriores que tais parâmetros influenciam de forma marcante a reologia [7] e a secagem [8] dos concretos.

Após a mistura, os concretos com diferentes teores de água foram moldados, sendo curados na temperatura de $8{ }^{\circ} \mathrm{C}$ por 72 horas, em ambiente de elevada umidade relativa $(\sim 100 \%)$. Utilizouse essa temperatura para cura, pois segundo a literatura, os concretos

Tabela I - Teores de água utilizados para o processamento do concreto e seus respectivos valores de distância de separação média entre as partículas da matriz (IPS) e entre os agregados (MPT)[6].

[Table I - Water content for the castables 'processing and the mean separation distance between matrix particles (IPS) and the coarse aggregates (MPT) [6].]

\begin{tabular}{cccc}
\hline \multicolumn{2}{c}{ Teores de água } & IPS $(\boldsymbol{\mu m})$ & MPT $(\boldsymbol{\mu m})$ \\
\cline { 1 - 2 }$\%$ - volume & \%-peso & & \\
\hline 13 & 3,82 & 0,0176 & 149,649 \\
\hline 15 & 4,51 & 0,0261 & 157,600 \\
\hline 17 & 5,24 & 0,0349 & 165,934 \\
\hline 20 & 6,39 & 0,0491 & 179,216 \\
\hline
\end{tabular}

apresentam maior probabilidade de explosão durante a secagem [9]. Nas amostras utilizadas para o ensaio de termogravimetria, termopares tipo $\mathrm{K}$ foram inseridos durante a moldagem $(1 \mathrm{~mm}$ abaixo da superfície e a meia altura do corpo-de-prova), para monitoramento da temperatura do concreto durante o teste.

\section{Medidas de permeabilidade e porosidade}

Avaliou-se a permeabilidade em amostras cilíndricas (altura (h) de $25 \mathrm{~mm}$ e diâmetro (d) de $75 \mathrm{~mm}$ ) secas a $8^{\circ} \mathrm{C}$ por 96 horas em sílica gel. Ajustou-se aos dados uma equação polinomial (equação de Forcheimer), permitindo obter assim a constante de permeabilidade não-Darciana $\left(\mathrm{k}_{2}\right)$ [5]. A porosidade total dos concretos foi obtida por meio do método de porosimetria de Arquimedes (amostras secas em sílica gel), utilizando querosene como líquido de imersão (norma ASTM C 20-87).

\section{Resistência mecânica}

A resistência mecânica foi calculada através do ensaio de compressão diametral (norma ASTM C 496-90), utilizando-se uma taxa de aplicação de carga constante (42 N/s). Realizou-se o teste em corpos-de-prova cilíndricos $(\mathrm{h}=\mathrm{d}=40 \mathrm{~mm})$ secos e úmidos (pós-cura) conforme condições descritas anteriormente.

\section{Termogravimetria}

Os ensaios termogravimétricos foram realizados em corposde-prova cilíndricos $(\mathrm{h}=\mathrm{d}=40 \mathrm{~mm})$ úmidos. Para isso, utilizouse um equipamento que consistia de um forno elétrico (potência de $1700 \mathrm{~W}$ ) acoplado a uma balança [10]. A coleta dos dados (tempo, temperatura do forno e amostra e massa) foi feita a cada $5 \mathrm{~s}$ e o aquecimento efetuado na taxa de $10^{\circ} \mathrm{C} / \mathrm{min}\left(25-800^{\circ} \mathrm{C}\right)$.

A perda de massa foi avaliada pelo parâmetro $\mathrm{W}_{\mathrm{d}}$ e sua derivada em relação ao tempo (taxa de secagem), definidos respectivamente nas equações A e B:

$$
\begin{aligned}
& \mathrm{W}_{\mathrm{d}}(\%)=100 \times\left(\frac{\mathrm{M}_{0}-\mathrm{M}}{\mathrm{M}_{\mathrm{f}}}\right) \\
& \frac{\mathrm{dW}_{\mathrm{d}}}{\mathrm{dt}}(\% / \mathrm{min})=\left(\frac{\Delta \mathrm{W}_{\mathrm{d}}}{\Delta \mathrm{t}}\right)
\end{aligned}
$$

$\mathrm{W}_{\mathrm{d}}$ indica a perda de água que ocorre durante o aquecimento em relação a massa final de concreto (seco); onde: M é a massa instantânea medida no tempo $\mathrm{t}_{\mathrm{i}}, \mathrm{M}_{0}$ é a massa inicial e $\mathrm{M}_{\mathrm{f}}$ é a massa final (devido a ocorrência de explosão dos corpos-deprova, $\mathrm{M}_{\mathrm{f}}$ foi calculado considerando-se a quantidade inicial de água adicionada durante o processamento dos concretos).

\section{RESULTADOS E DISCUSSÃO}

Na Fig. 1 é apresentada a constante de permeabilidade nãoDarciana $\left(k_{2}\right)$ em função da distância de separação média entre 
as partículas da matriz (IPS) e entre os agregados (MPT), para os concretos processados com diferentes quantidades de água. Verifica-se que essa constante relaciona-se de forma crescente com IPS, indicando aumentos expressivos de permeabilidade dos concretos. Tendo em vista que a elevação na quantidade de água tem como conseqüência o distanciamento entre as partículas da matriz (elevação de IPS - Tabela I), pode-se afirmar que após a secagem são geradas estruturas mais permeáveis em concretos com maiores teores de água, resultado este intuitivamente esperado.

Estudos anteriores realizados em concretos com diferentes granulometrias (teor de água constante) mostraram que o aumento no MPT foi responsável pela redução da permeabilidade, devido ao distanciamento entre regiões de maior porosidade que se formam na interface matriz agregado (ITZ - interfacial transition zone), e que favorecem a permeação de fluidos [8]. Embora fosse esperado redução de permeabilidade com o aumento de MPT, neste estudo observou-se justamente o contrário, ou seja, a elevação no MPT causou aumento na permeabilidade (Fig. 1). A variação no teor de água de $13 \%$ para $20 \%$ em volume causou elevação de aproximadamente $180 \%$ em IPS e de apenas $20 \%$ em MPT.

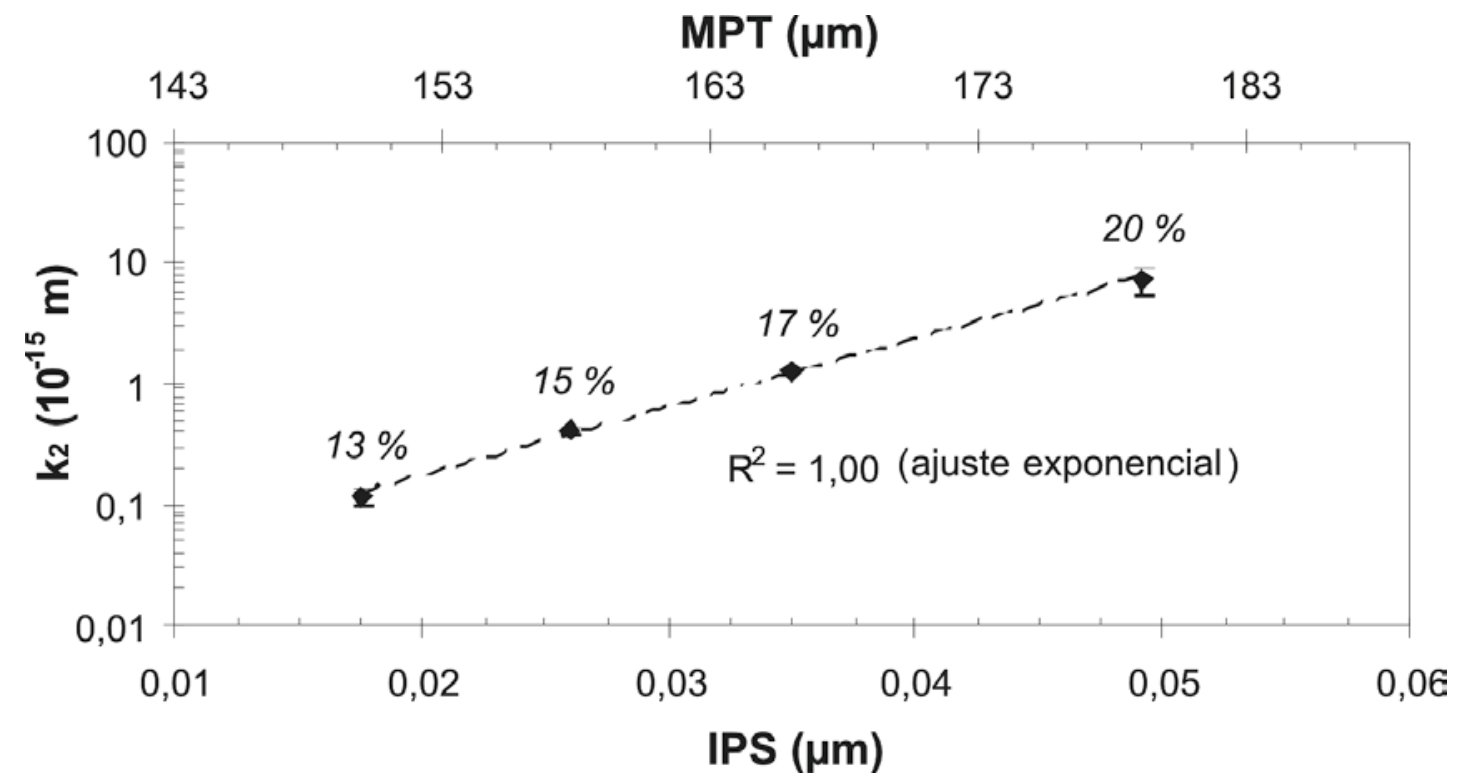

Figura 1: Constante de permeabilidade não-Darciana $\left(\mathrm{k}_{2}\right)$ em função da distância de separação entre as partículas da matriz (IPS) e entre o agregados (MPT), para os concretos processados com diferentes teores de água (13, 15, 17 e $20 \%$-vol).

[Figure 1: Non-Darcian permeability constant $\left(k_{2}\right)$ as a function of mean separation distance between matrix particles (IPS) and coarse aggregates (MPT), for castables prepared with different water content (13, 15, 17 and 20 vol-\%).]

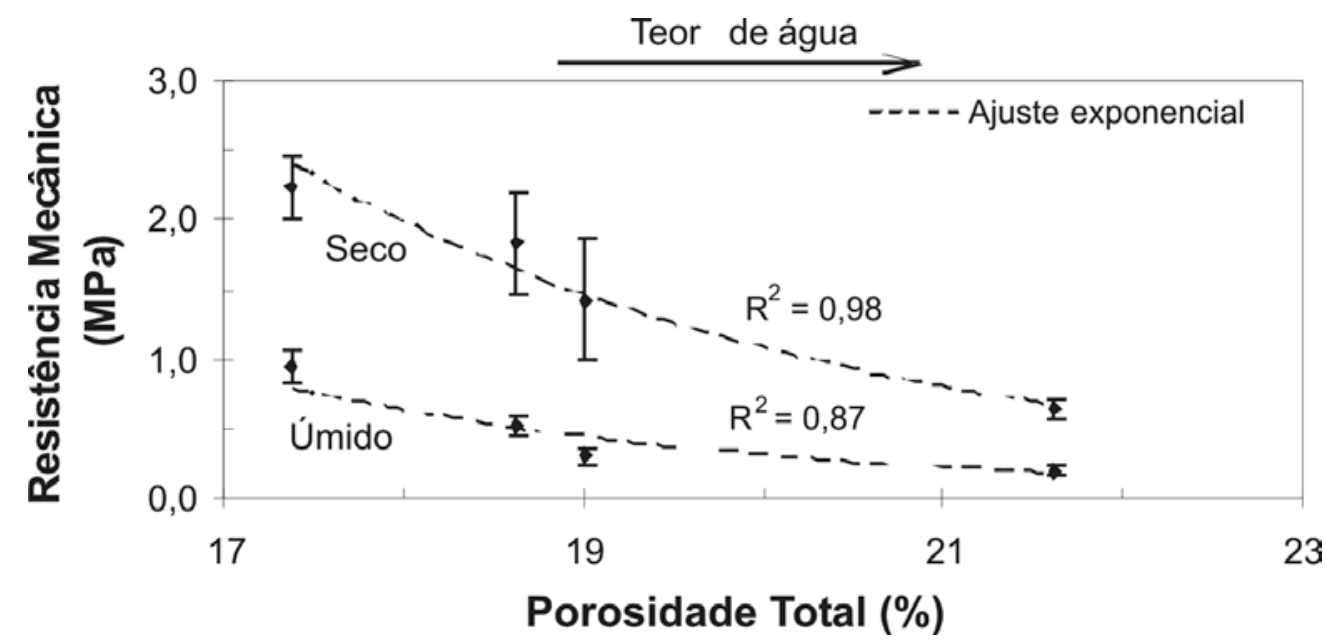

Figura 2: Resistência mecânica (compressão diametral) em função da porosidade total, para os concretos processados com diferentes teores de água (ensaiados úmidos e secos).

[Figure 2: Mechanical strength (diametral compression) as a function of total porosity, for castables prepared with different water content (wet and dry).] 


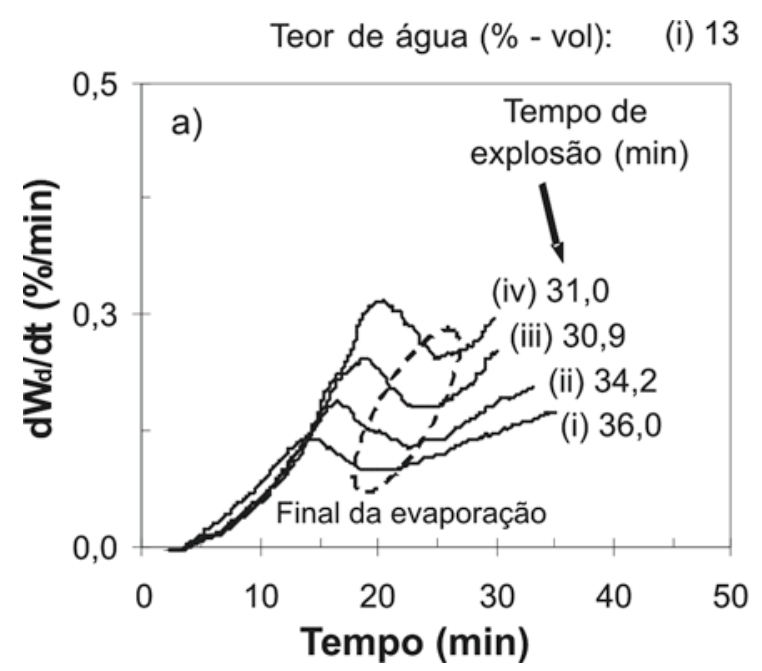

(ii) $15 \quad$ (iii) $17 \quad$ (iv) 20

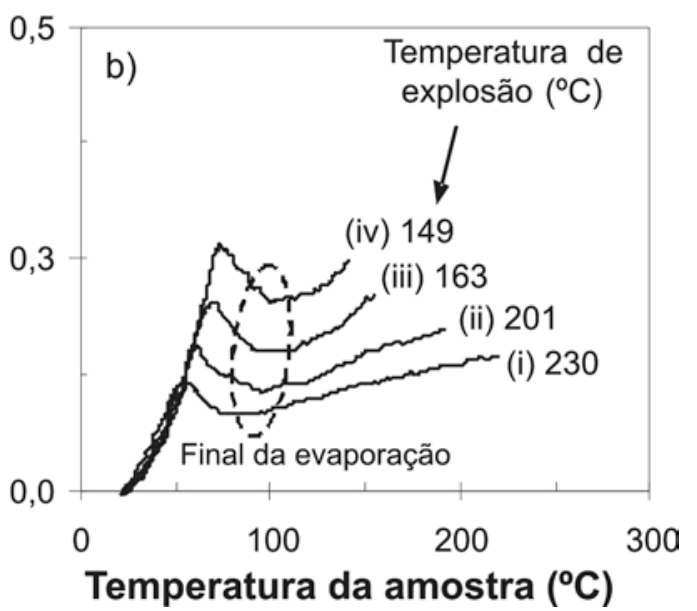

Figura 3: Taxa de perda de massa ( $\mathrm{dW}_{\mathrm{d}} / \mathrm{dt}$ ) em função do tempo (a) e da temperatura da amostra (b), para os concretos processados com diferentes teores de água.

[Figure 3: Drying rate $\left(d W_{d} d t\right)$ as a function of time (a) and sample temperature (b), for castables prepared with different water content.]

Isto mostra que apesar do acréscimo em MPT agir em favor da redução da permeabilidade, o aumento desta variável ocorreu principalmente devido a superior contribuição no IPS.

Observou-se também queda na resistência mecânica com a elevação no teor de água, em virtude do aumento da porosidade total dos concretos (Fig. 2). Esse ensaio, juntamente com o de permeabilidade, permitem identificar apenas as diferenças ocorridas após a saída da água (ensaios estáticos). No entanto, para uma análise mais realista do comportamento de secagem, também foram realizados ensaios termogravimétricos, que forneceram dados dinâmicos desse processo. Na Fig. 3 são apresentados estes resultados.

Em concretos refratários, no primeiro estágio de aquecimento $\left(25-100{ }^{\circ} \mathrm{C}\right)$, a água é removida do corpo cerâmico pelo processo de evaporação da água livre [10], e especificamente neste caso, pela decomposição de hidratos formados em baixas temperaturas de cura, como o $\mathrm{CaO} \cdot \mathrm{Al}_{2} \mathrm{O}_{3} \cdot 10 \mathrm{H}_{2} \mathrm{O}\left(\mathrm{CAH}_{10}\right)$ [9]. Este estágio tem seu término caracterizado pela inflexão na curva, sublinhado na Fig. 3.

Quando a temperatura do corpo cerâmico se aproxima de $100^{\circ} \mathrm{C}$, inicia-se o segundo estágio e o processo de secagem passa a ser comandado pela ebulição da água livre, sendo a formação de vapor intensificada pelo aumento de temperatura do forno [10]. Com o final da ebulição, inicia-se o terceiro estágio da secagem, a decomposição de hidratos mais resistentes a temperatura, como $\mathrm{Al}_{2} \mathrm{O}_{3} \cdot 3 \mathrm{H}_{2} \mathrm{O}\left(\mathrm{AH}_{3}\right)$ e $3 \mathrm{CaO} \cdot \mathrm{Al}_{2} \mathrm{O}_{3} \cdot 6 \mathrm{H}_{2} \mathrm{O}\left(\mathrm{C}_{3} \mathrm{AH}_{6}\right)[9,10]$. Apesar dos ensaios típicos de secagem em concretos refratários estarem caracterizados por estes três estágios principais, durante os experimentos foram observados somente a etapa de evaporação e parte da ebulição, devido a ocorrência de explosão dos corposde-prova testados.

Observa-se na Fig. 3-a que o aumento no teor de água tende a atrasar o final da evaporação em relação ao tempo. Embora a adição de água aumente a permeabilidade dos concretos, o tempo para o final da evaporação também é elevado, pois maior quantidade desse fluido deve ser extraído da amostra. Desta forma, foi utilizado um parâmetro normalizador que permitiu comparar os diversos concretos testados. Na Fig. 4-a é apresentada a taxa média de evaporação (TME) obtida através da equação $\mathrm{C}$.

$$
\operatorname{TME}(\% / \mathrm{min})=\frac{\% \text { água evaporada }}{\Delta t}
$$

onde TME é a taxa média de evaporação, \% água evaporada é a quantidade de água eliminada pelo processo de evaporação (em relação ao total de água presente) e $\Delta$ t é o tempo decorrido até o término deste estágio.

Como pode ser observado, a taxa média de evaporação se eleva com o conteúdo de água (Fig. 4-a). Este fato sugere que o aumento no teor de água pode levar os concretos a perder proporcionalmente mais água por evaporação em períodos menores, devido principalmente ao aumento de permeabilidade exposto na Fig. 1. Apesar disso, foi verificada uma maior tendência a explosão dos concretos com aumento no teor de água (redução da temperatura e do tempo de explosão - Fig. 3). Esse fato pode estar associado a duas razões principais:

1) Durante a etapa de evaporação o corpo-de-prova apresenta menor taxa de aquecimento que o forno, devido à transição de fase líquido-vapor da água (endotérmica) e à inércia do sistema forno/corpo-de-prova. Desta forma, é gerado um gradiente térmico entre a amostra e o forno, que pode ser intensificado pela elevação do teor de água, devido ao atraso do final da etapa de evaporação (maior quantidade de energia deve ser fornecida). Após a evaporação, os concretos processados com maior quantidade de água são expostos a um gradiente térmico mais elevado que as demais composições (Fig. 4-b), devido ao programa de aquecimento contínuo do forno. Com isso, o processo de secagem por ebulição é mais intenso e a transição de fase líquido/vapor ocorre com maior rapidez. No entanto, o corpo pode se tornar incapaz de eliminar 


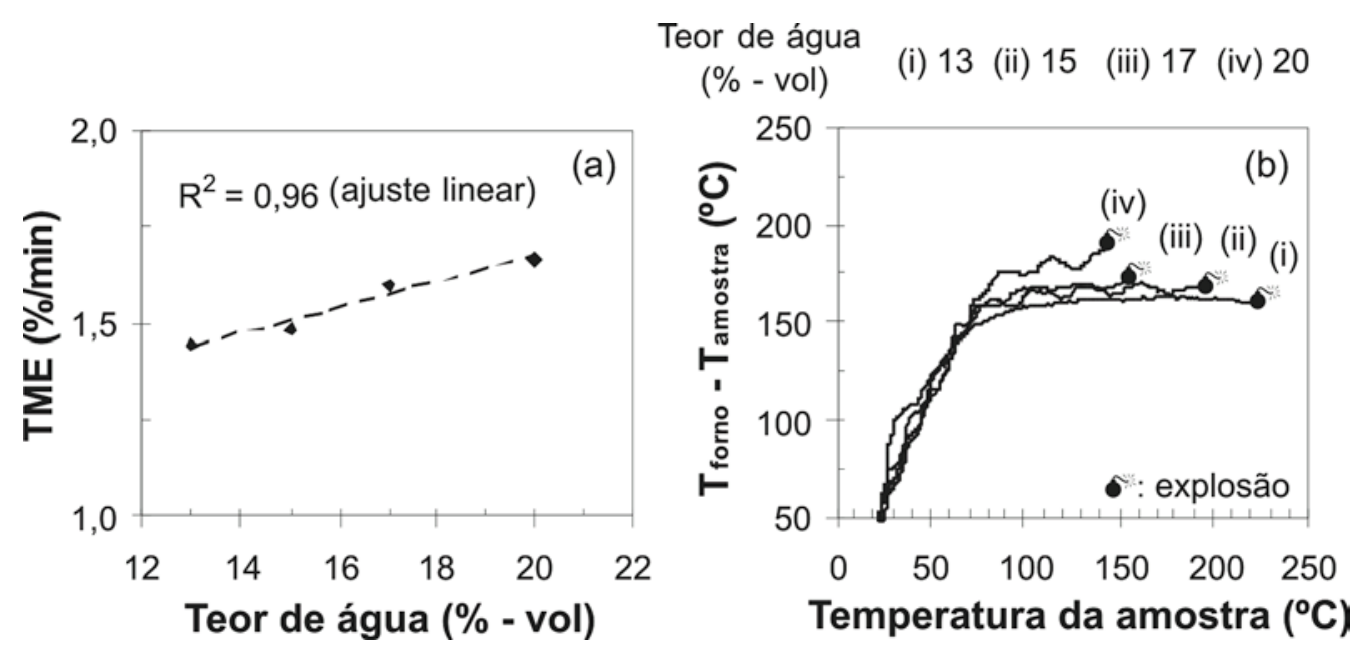

Figura 4: (a) Taxa média de evaporação (TME) em função do teor de água e (b) diferença de temperatura entre o forno e a amostra em função da temperatura da amostra, para os concretos processados com diferentes teores de água.

[Figure 4: (a) Mean evaporation rate (TME) as a function of water content and (b) the temperature gradient between furnace and sample ( $T_{\text {furnace }}-T_{\text {sample }}$ ) as a function of sample temperature, for castables prepared with different water content.]

o vapor com a mesma eficiência com que este é gerado, podendo levar a pressurização e conseqüente explosão.

2) Além do aumento no teor de água submeter as amostras a uma elevada formação de vapor após a evaporação (pressurização), a resistência mecânica também é reduzida (Fig. 2). Isso faz com que os corpos-de-prova apresentem menor probabilidade de resistir à pressão exercida pelo vapor d'água.

\section{CONCLUSÕES}

A elevação da quantidade de água adicionada durante a mistura dos concretos estudados foi responsável simultaneamente por:

- Aumento da permeabilidade e da porosidade;

- Redução da resistência mecânica;

- Redução da temperatura de explosão.

A adequação das características reológicas dos concretos em relação a mistura e transporte podem ser facilmente realizadas com o aumento no teor de água. Entretanto, foi mostrado que esse fato é acompanhado por várias outras desvantagens, que podem comprometer tanto o processo de secagem dos concretos (menor resistência a explosão), quanto suas características termomecânicas (redução da resistência mecânica) e de resistência a corrosão em altas temperaturas (maior porosidade e permeabilidade).

\section{AGRADECIMENTOS}

Os autores agradecem a FAPESP, ao CNPq, a Alcoa Alumínio S.A. e a Magnesita S.A. pelo suporte oferecido.

\section{REFERÊNCIAS}

[1] M. D. M. Innocentini, L. A. Nascimento, A. E. M. Paiva, V. C. Pandolfelli, B. A. Menegazzo, L. R. M. Bittencourt,
"Aluminum-containing refractory castables: Part I, Evaluation of hydrogen-gas generation", Am. Ceram. Soc. Bull. 82, 6 (2003) 45-49.

[2] R. Salomão, V. C. Pandolfelli, Drying Behavior of Polymeric Fiber-Containing Refractory Castables. Submetido à publicação no Journal of Technical Association of Refractories, Japan (2003). [3] R.G. Pileggi, A. R. Studart, C. Pagliosa Neto, V. C. Pandolfelli, "Caracterização reológica de concretos refratários bombeáveis", Cerâmica 46, 300 (2000) 176-180.

[4] R. Salomão, V. G. Domiciano, C. S. Isaac, R. G. Pileggi, V. C. Pandolfelli, "The mixing step and the permeability of polymeric fiber-containing refractory castables", Am. Ceram. Soc. Bull. 84, 1 (2003).

[5] M. D. M. Innocentini, A. R. Studart, R. G. Pileggi, V. C. Pandolfelli, "How PSD affects permeability of castables", Am. Ceram. Soc. Bull. 80, 5 (2001) 31-35.

[6] I. R. Oliveira, A. R. Studart, R. G. Pileggi, V. C. Pandolfelli, "Dispersão e empacotamento de partículas - Princípios básicos e aplicações no processamento cerâmico", Fazendo Arte Editorial (2000) 224 p.

[7] R. G. Pileggi, F. T. Ramal Jr., A. E. Paiva, V. C. Pandolfelli, "High performance refractory castable: Particle size design", Refractories Applications and News 8, 5 (2003) 17-21.

[8] M. D. M Inocentinni, R. G Pileggi, F. T. Ramal Jr, V. C. Pandolfelli, "Permeability and drying behavior of PSD-designed refractory castables". Am. Ceram. Soc. Bull. 82, 7 (2003) 1-6. [9] M. M. Akiyoshi, R. C. Morisco, M. D. M. Innocentini, V. C. Pandolfelli, "Temperatura de cura e sua Influência na resistência à explosão de concretos refratários de alta alumina", Cerâmica 48, 307 (2002) 126-130.

[10] M. D. M. Innocentini, F. A. Cardoso, M. M. Akyioshi, V. C. Pandolfelli, "Drying stages during the heat-up of highalumina, ultra low cement refractory castables", J. Am. Ceram. Soc. 86, 7 (2003) 1146-1148.

(Rec. 27/10/03, Ac. 28/11/03) 